\title{
Utilisation d'une fonction de forme pour l'établissement d'un tarif de cubage du chêne indigène
}

\author{
P. Balleux et H. Laudelout
}

Centre de Recherches Forestières, Unité des Eaux et Forêts, Université Catholique de Louvain, Route de la Fagne, B-6460 Chimay, Belgique

(reçu le 14 septembre 1988; accepté le 12 janvier 1989)

Résumé - Cette note propose l'utilisation d'un modèle paramétrique décrivant la variation de la surface de la section de la tige : $\mathbf{S}(h)$ du chêne indigène avec la hauteur $h$.

Cette relation a la forme: $S(h)=S_{0} / \sqrt{1+F h}$ (1); où $h$ est la hauteur comptée à partir du niveau du sol, $S_{0}$ est la surface de la section au niveau $h=0$ et $F$ est un paramètre de forme.

L'ajustement de cette relation à quelque 300 tiges de chêne a permis une étude statistique des deux paramètres $S_{0}$ et $F$. Le paramètre $S_{0}$ est bien corrélé avec la mesure directe de la section pour $h=0$. D'autre part, il existe une relation quadratique simple entre $S_{0}$ et la surface terrière.

La statistique du paramètre $F$ montre une distribution très asymétrique dont la valeur moyenne est de $0,56 \mathrm{~m}^{-1}$ et la médiane $0,47 \mathrm{~m}^{-1}$. Les variations du paramètre $F$ sont indépendantes de la circonférence de l'arbre.

L'utilité du modèle proposé repose essentiellement sur son emploi pour le calcul du volume $V$ de la tige jusqu'à une découpe $H: V / S_{0}=(2 / F)(\sqrt{1+F H}-1)$ (2); La comparaison entre les estimations individuelles du volume des tiges suivant l'équation (2) ou par quadrature numérique des sections mesurées, mètre par mètre, suivant la méthode de Simpson donne d'excellents résultats, pour la découpe "bûcheron". De bons résultats sont également obtenus pour la comparaison des volumes compris entre 0 et la découpe marchande, suivant le modèle de Huber.

En absence de données statistiques sur la valeur du paramètre $F$ dans l'échantillon de tiges étudié, il est aisé d'optimiser sa valeur par rapport au volume de référence et d'obtenir ainsi une mesure quantitative de la forme moyenne des tiges dans le peuplement.

volume - tarif - fonction de forme - chêne

Summary : Use of two-parameter taper function for determining the stem volume of native oak. A parametric model is presented which provides a good estimate of the taper function for the native oak.

This function is given by the following equation: $S(h)=S_{0} / \sqrt{1+F h} \quad(1)$; where $h$ is the stem height, $S_{o}$ is the value of the cross section at level $\mathrm{h}=0$ and $\mathrm{F}$ is a form parameter.

Non linear adjustement of this equation to the measured cross section areas of the stem allowed the determination of the statistics for the two parameters both of which have an obvious physical significance.

$S_{o}$ was satisfactorily correlated with the measured basal cross section, in spite of the inherent inaccuracy of girth or diameter taken at that level.

The parameter $F$ was distributed as shown by Fig. 3 with a mean value of $0,56 \mathrm{~m}^{-1}$ and a median of $0,47 \mathrm{~m}^{-1}$. 
The validity of the model is best established by comparing the definite integral from $\mathrm{O}$ to $\mathrm{H}$ of the following equation: $V / S_{0}=(2 / F)(\sqrt{1+F H}-1)$ (2); with the result obtained by Simpson's numerical integration with steps of one meter. The results of both methods were nearly identical (the residual standard deviation of the orthogonal regression was only $0,04 \mathrm{~m}^{3}$ ).

Using the model relies on an easily obtainable relationship between $S_{o}$ and girth at 1,3 $\mathrm{m}$, the stem height and either the median or average value of the $F$ parameter. It was found that calculating the $S_{0}$ parameter by a linear regression on the stem cross section at 1,3 $\mathrm{m}$ led to a serious overestimate of the volume of the smaller stems in the range studied i.e. between 40 and $100 \mathrm{~cm}$ circumference at $1,3 \mathrm{~m}$. Using a quadratic regression equation gave satisfactory results.

Optimizing the value of parameter $F$ in equation (2) as shown in Fig. 4 by minimizing the root mean square of the differences between the volumes calculated according to Simpson's method and equation (2) supplies an estimate of the average of the form parameter which may be of use for characterizing the stem profile of the studied sample.

stem volume - taper function - oak

\section{Introduction}

L'établissement d'un volume de référence aussi exact que possible est le préalable indispensable à l'élaboration d'un tarif de cubage pour une essence dans une région donnée.

Nous nous proposons d'exposer et de justifier un modèle paramétrique simple permettant à la fois l'établissement du volume de référence et le calcul pratique du cubage par une seule formule ne comprenant que deux paramètres de signification physique évidente.

Cette technique de calcul a été appliquée au chêne indigène (Quercus robur L. $=Q$. pedunculata Ehrh. et $Q$. petraea Liebl. = Quercus sessilis Ehrh. = $Q$. sessiliflora Salisb. et leurs hybrides) constituant la grande majorité de la futaie des peuplements forestiers de la Fagne de Chimay, de la Thiérache et de l'Avesnois, conduits en taillis de charme sous futaie de chêne.

La valeur marchande de cette essence et l'irrégularité de sa géométrie justifient, à des titres très différents, une approche nouvelle à la fois du calcul du volume de référence et de l'élaboration d'un tarif de cubage.
En général, les méthodes actuelles de calcul du volurne de référence impliquent, à partir des mesures de terrain, deux types de calcul:

- le calcul de la surface de la section droite de la grume (abrégé en "section", ciaprès) à divers niveaux, soit à partir de la circonférence, soit à partir d'un ou de deux diamètres.

- le calcul du volume compris entre le niveau zéro et un niveau de découpe $\mathrm{H}$ à partir des valeurs de la section à un certain nombre de niveaux entre 0 et $\mathrm{H}$, au minimum 3, comme dans la méthode de I'Inventaire Forestier National.

Dans ce qui suit, nous ne nous préoccuperons pas de l'erreur associée à la première étape, sinon pour mentionner qu'elle existe et est liée à l'irrégularité de la géométrie de l'essence et qu'elle constitue une justification partielle de l'opération de lissage décrite ci-dessous.

En ce qui concerne le second calcul, il existe deux approches possibles : la première, la plus courante, consiste à considérer les mesures de la section effectuées à divers niveaux comme les évaluations d'une fonction $S(h)$ dont l'intégrale définie de 0 à $H$ est égale au volume compris entre ces niveaux. 
Ceci revient donc au problème de l'intégration d'une fonction $\mathrm{S}(h)$ qui n'est connue que par un tableau de ses valeurs $S\left(h_{i}\right)$ en un certain nombre de points de l'intervalle d'intégration. La solution de ce problème est représenté par les méthodes d'intégration numérique de Newton-Cotes (cf. par exemple Gerald et Wheatley 1984).

En bref, ces techniques consistent à approximer une intégrale définie I par une somme :

$$
I=\sum A_{i} S\left(h_{i}\right)
$$

où les $A_{i}$ sont des constantes.

La méthode la plus simple consiste à prendre pour les $A_{i}$ la largeur des intervalles au centre desquels la fonction a été évaluée. C'est la méthode des rectangles qui est à la base des tables dendrométriques de Dagnelie et al. (1976).

Si l'on choisit de relier les points d'évaluation de la fonction par des segments de droite, on applique la méthode des trapèzes qui est une approximation de la méthode de cubage par billons tronconiques utilisée par Dagnelie et al. (1985).

Une autre méthode consiste à réunir trois évaluations de la fonction sur deux intervalles égaux par des arcs de parabole, suivant la méthode d'interpolation de Newton, ce qui donne la méthode de Simpson au 1/3. Cette méthode est considérée par les forestiers comme une méthode de référence et sera utilisée comme telle dans ce travail.

Il est clair que la qualité de la quadrature numérique dépendra essentiellement de l'exactitude de l'évaluation de $S(h)$ et du nombre d'évaluations effectuées dans l'intervalle d'intégration, c'est-à-dire du pas de l'intégration numérique ou, en termes forestiers, de la "longueur des billons".

L'autre approche consiste à lisser les évaluations de la fonction $S(h)$ par une relation telle que son intégration soit aisée. La technique du lissage des points expérimentaux présente incontestablement des dangers comme le signalent pratiquement tous les manuels d'analyse numérique qui en traitent. La meilleure analyse du problème nous semble être celle de Press et al. (1986) que les auteurs résument en : "smoothing is art, not science"

Nous nous proposons d'étudier dans cette note une technique de lissage des estimations de la section d'une tige à diverses hauteurs, d'en présenter la validation, puis l'application à la construction d'un tarif de cubage.

\section{Méthodes expérimentales et numé- riques}

L'étude a porté sur quelque 300 chênes des espèces citées ci-dessus, y compris quelques hybrides et indéterminés.

Le site des diverses chênaies étudiées se trouvait exclusivement à la Fagne de Chimay, bien que des peuplements identiques puissent être trouvés sur une bien plus grande surface de part et d'autre de la frontière entre la France et la Wallonie.

Les mensurations ont été effectuées sur des arbres abattus. On sait (Bouchon, 1984) que cette façon de faire peut introduire un biais, sauf si la distribution des arbres abattus reflète suffisamment bien celle du peuplement. La Fig. 1 montre la distribution d'un peuplement type de la Fagne de Chimay et celle des délivrances correspondantes pour une coupe de $30 \mathrm{Ha}$. L'utilisation du graphique de probabilité justifie l'échantillonnage utilisé, quelles que soient les conséquences du parallélisme des distributions observées au point de vue sylvicole.

Les mensurations de grosseur ont été relevées au compas forestier, suivant 


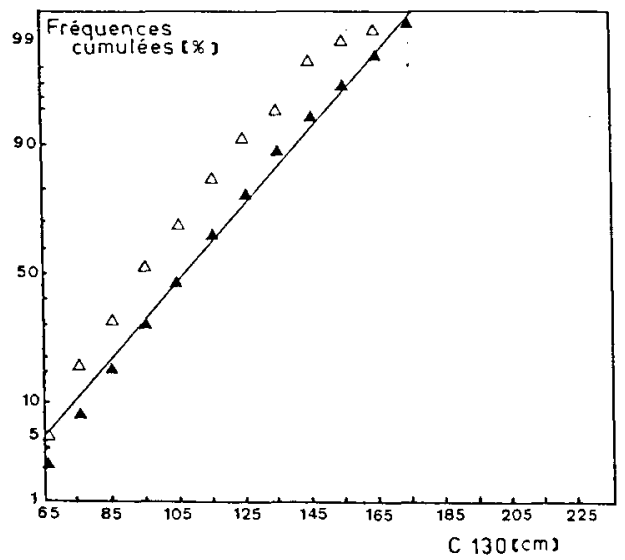

Fig. 1. Graphique de probabilité des fréquences cumulées des circonférences dans un peuplement de $30 \mathrm{Ha}$, et des délivrances effectuées.

deux diamètres perpendiculaires. La circonférence a été mesurée à 1,30 et $1,50 \mathrm{~m}$ de hauteur ainsi qu'à la découpe "bûcheron", à la mi-hauteur de cette découpe, à la moitié de la hauteur totale, à la découpe marchande et à la moitié de la hauteur de cette découpe. Les diamètres ont été mesurés à ces niveaux et de mètre en mètre jusqu'au dernier billon avant la découpe "bûcheron".

II est évidemment possible que les mihauteurs coïncident ou s'approchent des hauteurs entières en $\mathrm{m}$. Dans ce cas, elles n'ont pas été prises en compte, comme on peut le voir à la Fig. 2 . On trouvera plus loin une statistique des hauteurs mesurées et des circonférences correspondantes pour les découpes "bûcheron" et marchande.

Une fiche de relevé a été établie de façon à faciliter au maximum la prise de données sur le terrain, mais aussi la transcription de celles-ci sur ordinateur. Des programmes ont été écrits pour l'entrée des données sur un mode conversationnel. Après vérification des transcriptions, d'autres programmes effectuaient les cal- culs numériques, statistiques ou d'ajustement non linéaire sur l'ensemble des données fournies pour chaque grume soit environ de 50 à 70 nombres, suivant la longueur de la grume.

Les calculs portaient sur la détermination de la section aux divers niveaux à partir de la moyenne géométrique des diamètres mesurés à ce niveau, du volume de référence de la grume suivant huit méthodes différentes, du calcul de divers paramètres de forme et enfin, de l'ajustement des données au modèle décrit cidessous.

Le langage utilisé pour ces programmes de calcul était le RPL, un langage dérivé du $C$, qui fait partie d'un logiciel le RS/ 1 de la BBN Software Products Corporation. Tant le langage que le logiciel nous ont semblé parfaitement adaptés à ce travail.

Le nombre de mesures effectuées peut sembler exagéré et il l'est effectivement, mais il se justifie par la nécessité de valider les résultats de calcul du modèle par les résultats de méthodes classiques exécutées avec le maximum de soin.

\section{Résultats}

Le choix d'une relation $S(h)$ destinée à lisser les observations des valeurs de la section s'est fait en s'inspirant des critères suivants :

- La relation doit comprendre un nombre de paramètres aussi petit que possible et chacun d'eux doit avoir une signification physique évidente.

- La fonction $\mathbf{S}(h)$ admet une primitive qui permet le calcul de l'intégrale définie entre deux hauteurs données c'est-à-dire du volume de la tige compris entre les deux niveaux. 
- La relation $S(h)$ doit pouvoir être validée par comparaison entre le résultat de son intégration et le résultat du calcul du volume fait de façon indépendante sur les données brutes c'est à dire non-lissées.
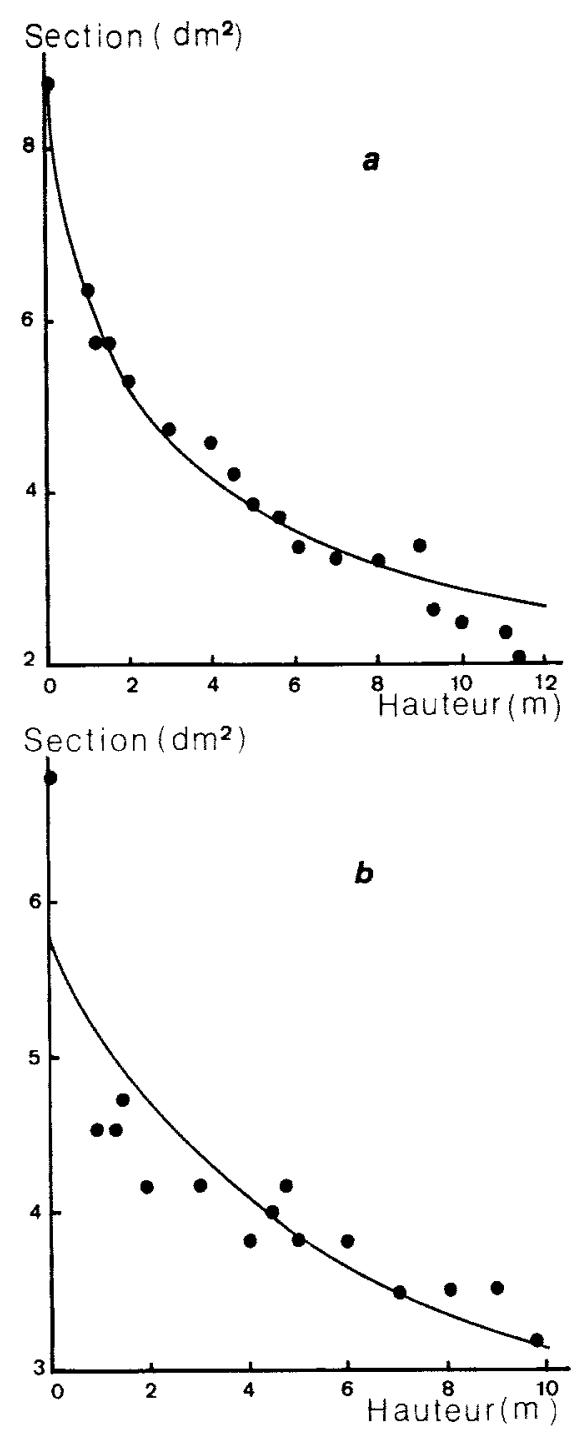

Une fonction qui semble répondre à ces critères est :

$$
S(h)=S_{0} / \sqrt{1+F h}
$$
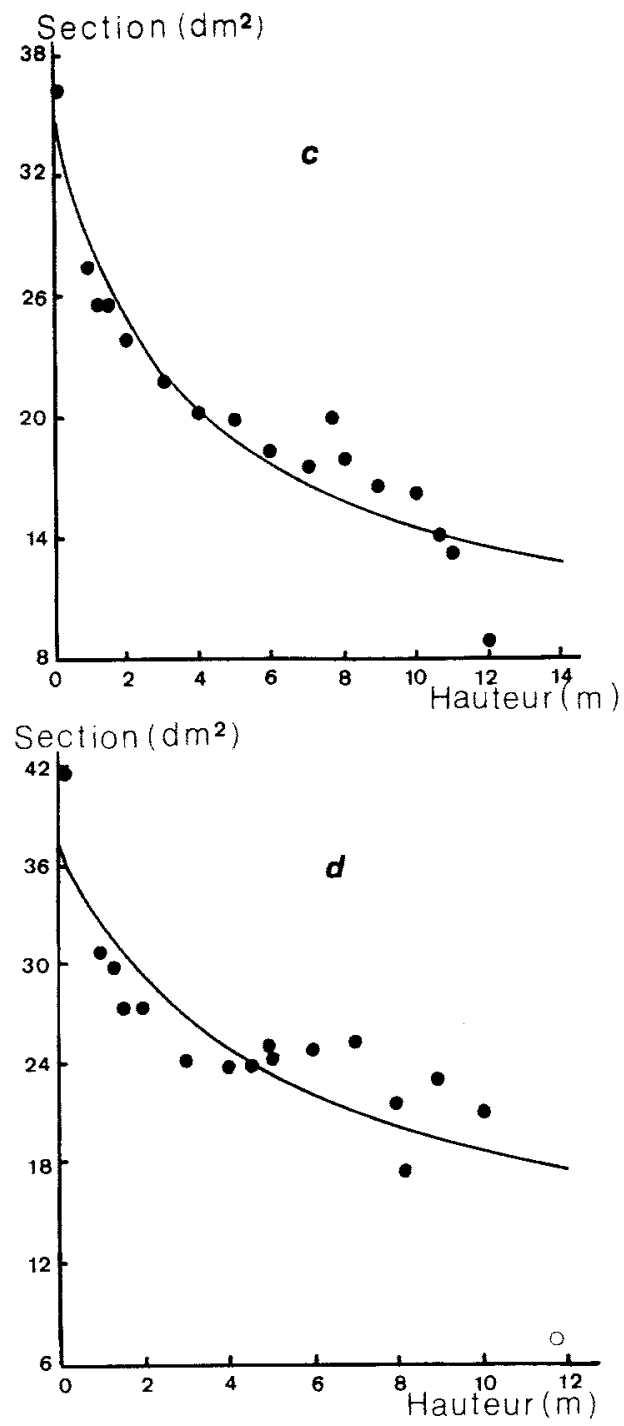

Fig. 2. Exemples de lissage des mesures de la section, en fonction de la hauteur, suivant le modele de l'équation (1), pour deux baliveaux de chêne $(a$ et $b$ ) et pour deux anciens ( $c$ et $c$ ) à deux niveaux extrémes de qualité de l'ajustement du modèle. 
où $S_{0}$ est la section au niveau du sol $(h=0)$ et le paramètre $F$ est un paramètre de forme qui exprime l'effet de $h$ sur la diminution de $S$, dans le cas d'un cylindre, on a évidemment $F=0$.

Comme elle ne contient que deux paramètres dont la signification est évidente, le premier critère est satisfait. II en est de même du second puisque l'on a, par intégration de $\mathrm{S}(h)$ de 0 à $\mathrm{H}$.

$$
V / S_{0}=(2 / F)(\sqrt{1+F H}-1)
$$

Le lissage des valeurs de la section mesurées à des hauteurs allant de 0 à la découpe «bûcheron" se fait généralement de façon satisfaisante comme le montre la Fig. 2, dans laquelle nous avons regroupé 4 exemples de courbes théoriques dérivés de (1) à partir des points expérimentaux. Les quatre figures ont été choisies de façon à illustrer deux types d'ajustement : bon ( $a$ et $b$ ) et médiocre ( $c$ et $d$ ) exécutés sur deux classes de circonférence : 80 et $190 \mathrm{~cm}$.

De façon plus précise, les statistiques des quatre ajustements figurant à la Fig. 2 sont présentées au Tableau I qui reprend la valeur des deux paramètres $S_{0}$ et $F$, leur erreur standard et l'écart quadratique moyen à la courbe théorique.

La statistique du paramètre $S_{0}$ ne présente guère d'intérêt pour le problème qui nous occupe. II était toutefois intéressant de vérifier que ce paramètre possédait effectivement le sens qui lui est attribué.

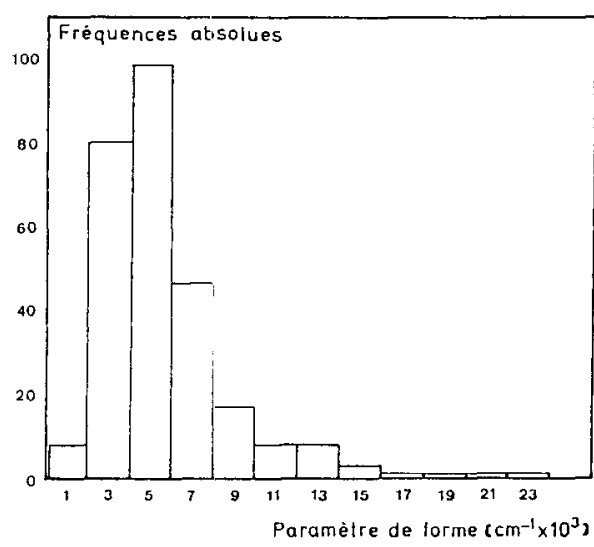

Fig. 3. Histogramme des fréquences absolues du paramètre de forme.

En calculant la régression du paramètre $S_{0}$ en fonction de la valeur effectivement mesurée de la section au ras du sol, on trouve pour les coefficients de la régression linéaire, $0,959 \pm 0,006$ pour la pente et $-0,15 \pm 0,22$ pour l'ordonnée à l'origine.

L'accord est aussi bon que possible compte tenu de la surestimation qui affecte la mesure directe en raison de l'empattement. Le paramètre $S_{0}$ est donc bien une indication de ce que serait la section basale si la décroissance constatée sur la tige se continuait jusqu'à la base, sans être modifiée par l'empattement.

Le paramètre de forme calculé pour chacune des tiges étudiées possède un domaine de variation considérable autour d'une valeur moyenne de $0,56 \pm 0,02 \mathrm{~m}^{-1}$

Tableau I. Statistiques de 4 ajustements typiques de l'équation (1) à 4 arbres.

\begin{tabular}{llllll}
\hline$N^{\circ}$ Arbre & C130 $(\mathrm{m})$ & $H(\mathrm{~cm})$ & $F\left(\mathrm{~m}^{-1}\right)$ & So $\left(\mathrm{dm}^{2}\right)$ & Ecart quadratique moyen \\
\hline Q263 & 89 & 11,2 & $0,91 \pm 0,08$ & $8,8 \pm 0,3$ & 0,80 \\
Q235 & 82 & 9,0 & $0,25 \pm 0,05$ & $5,7 \pm 0,3$ & 0,15 \\
Q112 & 192 & 12,0 & $0,49 \pm 0,08$ & $34,8 \pm 1,7$ & 4,30 \\
Q131 & 193 & 10,1 & $0,32 \pm 0,08$ & $34,5 \pm 2,7$ & 13,30 \\
\hline
\end{tabular}


et d'une médiane de $0,47 \mathrm{~m}^{-1}$. L'asymétrie de sa distribution est illustrée par la Fig. 3.

L'utilisation des deux paramètres $S_{0}$ et $F$ calculés par lissage des valeurs de $S(h)$ permet le calcul du volume individuel des tiges à partir de la formule (2) où l'on introduit pour chaque tige la hauteur $\mathrm{H}$ correspondant à une découpe donnée.

Un premier exemple de comparaison entre le volume Simpson et les résultats du calcul suivant l'équation (2) avec deux paramètres propres à chaque tige et obtenus par lissage des données sur la section se rapporte au volume du niveau 0 à la découpe «bûcheron". Pour l'échantillon étudié, cette découpe se fait à une hauteur de 6,2 à 18,5 $\mathrm{m}$ avec une moyenne de 12,7 $\mathrm{m}$ et un écart-type de $2,0 \mathrm{~m}$. La médiane et la moyenne sont identiques, ce qui reflète les résultats de la Fig. 1. La circonférence de la tige à ce niveau est de $89 \mathrm{~cm}$ en moyenne avec un écart-type de $32 \mathrm{~cm}$. Cette découpe peut être comparée à la hauteur totale moyenne des arbres de l'échantillon, soit $20,8 \mathrm{~m}$ avec un écarttype de 2,2 $\mathrm{m}$. Elle représente donc $60 \%$ de la hauteur totale.

On trouve pour le coefficient angulaire de la régression orthogonale entre le volume suivant l'équation (2) et celui de la méthode Simpson la valeur de 0,9987 , et pour l'ordonnée à l'origine $0,004 \mathrm{~m}^{3}$. L'écart-type résiduel à cette régression est de $0,042 \mathrm{~m}^{3}$.

Un second exemple porte sur le calcul du volume marchand défini par la formule de Huber. La hauteur marchande dans l'échantillon étudié allait de 4,5 à 15,0 m avec une moyenne de $8,9 \mathrm{~m}$ et un écarttype de $2 \mathrm{~m}$, soit $70 \%$ de la découpe "bûcheron" ou $40 \%$ de la hauteur totale.

On a trouvé pour le coefficient angulaire de la régression orthogonale la valeur de 0,9898 et pour l'ordonnée à l'origine $-0,011 \mathrm{~m}^{3}$. L'écart-type résiduel est de $0,12 \mathrm{~m}^{3}$.
Comme le calcul du volume marchand suivant Huber est d'une extrême simplicité, l'accord entre les deux calculs du volume est aussi bon qu'il était raisonnable de l'espérer.

Comme l'intégrale de la fonction de défilement entre deux niveaux donne des résultats pratiquement identiques à ceux que donnent deux méthodes indépendantes pour le calcul du volume, on peut considérer que le troisième critère énoncé ci-dessus (à savoir la validation du modèle par un calcul indépendant) est réalisé.

\section{Discussion}

Utilisation de lintégrale de la fonction de forme comme tarif de cubage

Dans ce qui précède, les deux paramètres de l'équation (2) étaient obtenus par l'ajustement non linéaire de l'équation (1) aux mesures de la section le long de chacune des tiges. Si l'on désire utiliser l'équation (2) pour confectionner un tarif de cubage à double entrée, il est nécessaire de trouver la relation entre l'une des entrées classiques (par exemple, la circontérence à $1,30 \mathrm{~m}$ ) et le paramètre $S_{0}$; et d'autre part, de déterminer quelle valeur du paramètre $F$ fera de celui-ci une bonne variable explicative du volume de référence mesuré.

II n'y a guère de difficulté à mettre en évidence une relation entre $S_{0}$ et la circonférence mesurée à $1,30 \mathrm{~m}$.

On trouve une relation linéaire apparemment satisfaisante entre $S_{0}$ et la surface terrière (ST $=\mathrm{C}_{130^{2}} / 4 \Pi$ ) de la forme :

$$
S_{0}=(2,04 \pm 0,4)+(1,194 \pm 0,017) \times S T
$$

et une relation quadratique :

$$
\begin{aligned}
S_{0}= & (-0,849 \pm 0,6)+(1,522 \pm 0,05) \\
& \times \text { ST }-(0,0063 \pm 0,001) \times \mathrm{ST}^{2}
\end{aligned}
$$


Mais la régression linéaire surestime la valeur du paramètre $S_{0}$ dans le domaine des petites circonférences $\left(C_{130}<1,20 \mathrm{~m}\right)$, ce qui n'est pas le cas pour la régression quadratique.

Le calcul de $S_{0}$ à partir de $C^{2}{ }_{130} / 4 \pi$ est plus accessible et plus fiable que son estimation à partir de la section basale en raison des difficultés de mesure de circonférence ou de diamètre à ce niveau et surtout du fait qu'il ne s'agit pas là d'une mesure courante.

En ce qui concerne le second paramètre, deux solutions sont en principe possible pour son introduction dans le tarif de cubage. La première consiste à introduire dans la formule de calcul la valeur médiane de $F$ trouvée pour l'échantillon soit $0,47 \mathrm{~m}^{-1}$.

En utilisant ce calcul pour une hauteur de tige égale à la découpe «bûcheron» (hauteur dont les statistiques ont été données ci-dessus) pour un domaine de circonférence allant de 45 à $295 \mathrm{~cm}$, on trouve pour la régression orthogonale donnant le volume de référence suivant Simpson en fonction du volume calculé par le modèle : 1,04 pour la pente et $-0,08 \mathrm{~m}^{3}$ pour l'ordonnée à l'origine. L'écart-type résiduel est $0,18 \mathrm{~m}^{3}$.

Ecaft type residuel $\left(\mathrm{m}^{3}\right)$

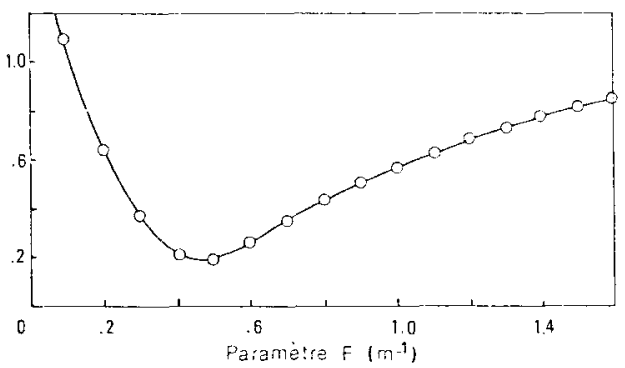

Fig. 4. Optimisation du parametre de forme $F$ par la minimisation de la racine carrée de l'écart quadratique moyen entre le volume calcule suivant l'equation (2) et le volume suivant Simpson.
La seconde technique consiste, en quelque sorte, à calquer la démarche suivie pour l'établissement des coefficients d'un modèle numérique du volume par régression multiple en fonction des variables $\mathrm{C}$ et $\mathrm{H}$ et de leur produits. Pour le tarif de cubage décrit ici, l'optimisation se limite à celle du seul paramètre $F$, puisque le paramètre $S_{0}$ est calculé comme dit ci-dessus. La valeur du paramètre $F$ est celle qui minimise l'écart quadratique moyer entre le volume Simpson de chaque tige et celui qui est calculé en utilisant une valeur donnée du paramètre $\mathrm{F}$ dans le tarif. L'écart quadratique moyen correspondant à 16 valeurs successives du paramètre $F$ est mis en graphique à la Fig. 4.

II est clair qu'un minimum de cet écart valant environ $0,19 \mathrm{~m}^{3}$ s'observe pour une valeur médiane de F. La Fig. 4 fournit aussi une estimation de la sensibilité du tarif à l'exactitude de la détermination du paramètre $F$ : l'écart quadratique moyen diminue très rapidement à partir de $F=0$ jusqu'à sa valeur médiane puis augmente plus lentement ensuite.

\section{Conclusion}

Le modèle de calcul qui a été utilisé ici et dont la validation a donné des résultats satisfaisant est intermédiaire entre les modèles purement numériques tels que ceux qui ont été présentés par Dagnelie et al. $(1975,1985)$ et des modèles déterministes liés à la morphogénèse d'un système vivant dont Bouchon (1984 p. 8) a ébauché la systématique.

L'avantage du modèle paramétrique consiste en ce qu'il permet une estimation pour un peuplement donné d'un paramètre unique, caractéristique du profil moyen de l'arbre dont il mesure l'effet sur le volume de la tige. 
II est clair que ce paramètre est dissimulé dans le modèle numérique issu de la régression multiple. C'est d'ailleurs là le principe de la méthode utilisée par Demaerschalk $(1971,1973)$ pour extraire d'un modèle numérique du volume une équation empirique décrivant la variation du diamètre avec la hauteur et inversement.

On peut donc résumer la conclusion de cette étude comme suit :

- Le modèle représenté par l'équation (2) permet le calcul du volume de référence d'une tige avec une précision comparable à celle de la méthode de Simpson pour la longueur totale d'une grume sur le parterre de la coupe et une estimation du volume marchand pratiquement égale à celle du volume Huber.

- Le modèle permet la confection d'un tarif de cubage à double entrée $C_{130}$ et la hauteur de découpe, aisément programmable, ce qui remplace le recours aux tables.

- En l'absence d'une statistique des valeurs de $F$, l'optimisation de ce paramètre unique fournit une estimation de sa valeur médiane caractéristique de la forme des tiges du peuplement. C'est là une indication quantitative, qui est peutêtre utile, quelle que soit sa dispersion statistique, laquelle ne fait probablement que traduire l'hétérogénéité d'un peuplement de chêne conduit en taillis-sousfutaie, dans les conditions de sol et de climat de l'échantillon étudié.

\section{Remerciements}

Les auteurs remercient vivement Monsieur le Professeur P. Dagnelie (Gembloux), Monsieur R.B. Chevroux Ingénieur en Chef du GREF et Monsieur J. Bouchon, Directeur de $\mathrm{Re}-$ cherches, pour une lecture attentive de la première version du manuscrit et des suggestions destinées à en améliorer la clarté de la présentation.

\section{Bibliographie}

Bouchon J. (1984) Les tarifs de cubage. E.N.G.R.E.F., Nancy. 57 pp.

Dagnelie P., Rondeux J. \& Thill A. (1976) Tables Dendrométriques. Les Presses Agronomiques de Gembloux. 128 pp.

Dagnelie P., Palm R., Rondeux. J. \& Thill A. (1985) Tables de cubage des arbres et des peuplements forestiers. Les Presses Agronomiques de Gembloux. 148 pp.

Demaerschalk J.P. (1971) Taper equation can be converted to volume equations and point sampling factors. Forestry Chron. 352-354

Demaerschalk J.P. (1973) Integrated systems for the estimation of tree taper and volume. Can. J. For. Res. 3, 90-94

Gerald C.F. \& Wheatley P.O. (1984) Applied numerical analysis. 3rd Edition. Addison-Wesley Publ. Co 579 pp.

Pardé J. et Bouchon J. (1988) Dendrométrie. Deuxième édition. E.N.G.R.E.F., Nancy. 328 pp.

Press W.H., Flannnery B.P., Teukolsky S.A. \& Vetterling W.T. (1986) Numeral recipes. The art of scientific computing. Cambridge University Press. 818 pp. 